\title{
Prognosticating ovarian reserve by the new ovarian response prediction index
}

\author{
Apoorva Tak ${ }^{1 *}$, Madhubala Chauhan', Bharti Tak ${ }^{2}$
}

\begin{abstract}
${ }^{1}$ Department of Obstetrics and Gynecology, Dr Madhubala Chauhan RNT Medical College, Udaipur, Rajasthan, India ${ }^{2}$ Department of Obstetrics and Gynecology, Bharti Tak SN Medical College, Jodhpur, Rajasthan, India
\end{abstract}

Received: 18 November 2017

Accepted: 18 December 2017

\section{*Correspondence:}

Dr. Apoorva Tak,

E-mail: drapoorvatak90@gmail.com

Copyright: (c) the author(s), publisher and licensee Medip Academy. This is an open-access article distributed under the terms of the Creative Commons Attribution Non-Commercial License, which permits unrestricted non-commercial use, distribution, and reproduction in any medium, provided the original work is properly cited.

\section{ABSTRACT}

Background: Knowledge of patient's potential ovarian response can help individualise the medication dosage and thus prevent adverse effect of excessive ovarian response and, decrease the rates of cancelled cycles, overall it will help to improve the cost benefit ratio of ovarian stimulation regimes. In addition to age, several clinical, endocrine and USG markers and dynamic tests have been proposed for the prediction of ovarian response to stimulation. Among these use of AMH levels and AFC are of particular interest However, despite the predictive power that each marker for the ovarian response may individually have, all these markers have errors associated with their estimation. So, prediction of ovarian response using a single biomarker may not be sufficient for formulation of a precise treatment plan.

Methods: This study includes 50 patients undergoing ivf cycle. All patients satisfied the following criteria; age <or equal to 39 yrs, BMI between 20-30, regular menstrual cycles, both ovaries present, no history of ovarian surgery, no severe endometriosis and no evidence of endocrine disorders. The only exclusion criteria was the presence of ovarian cysts as assessed by TVS.

Results: The results showed significant correlation between the number of obtained follicles and the number and maturity of collected oocytes. In addition, the results using the ORPI were always better than those obtained using other predictive factors (AFC, AGE and AMH) separately.

Conclusions: The present study reinforces the ORPI, which is a simple 3 variable index that exhibits an excellent ability to predict low and excessive ovarian response. ORPI might be used to improve the cost benefit ratio of ovarian stimulation regimens by guiding the selection of medication and by tailoring the doses and regimens to the actual needs of patients.

Keywords: Individualised controlled ovarian stimulation, Ovarian response prediction index, Ovarian response, AMH levels

\section{INTRODUCTION}

There are various ovulation induction protocols used in IVF cycles to induce multi follicular development which increases the number of embryos for selection and transfer. $^{1}$ Knowledge of patient's potential ovarian response can help individualise the medication dosage and thus prevent adverse effect of excessive ovarian response and, decrease the rates of cancelled cycles, overall it will help to improve the cost benefit ratio of ovarian stimulation regimes. The first indicator of ovarian reserve taken into account is patient's age Although the number and quality of oocytes both decrease with age the reproductive potential varies drastically in women of 
similar age. In fact in addition to age, several clinical, endocrine and USG markers and dynamic tests have been proposed for the prediction of ovarian response to stimulation. $^{2}$ Among these use of AMH levels and AFC are of particular interest. However, despite the predictive power that each marker for the ovarian response may have individually, all these markers have errors associated with their estimation. So prediction of ovarian response using a single biomarker may not be sufficient for formulation of a precise treatment plan. ${ }^{3}$ The ORPI (ovarian response prediction index) which is based on all 3 parameters $\mathrm{AMH}, \mathrm{AFC}$, and age can be evaluated as a better parameter to evaluate ovarian response.

ORPI [AMH (ng/ml) x AFC (2-9 mm)/ patient age] exhibited an excellent ability to predict a low ovarian response and a good ability to predict retrieval of $>4$ MII oocytes, the occurrence of excessive ovarian response and occurrence of pregnancy in infertile women. ${ }^{4}$

The ORPI might be used to improve the cost benefit ratio of ovarian stimulation regimes by guiding the selection of medications and by modulating the doses and regimens according to patient's need. The predictors of ovarian reserve also serve to predict the FSH dosage for ovarian stimulation.

The management of poor ovarian response remains one of the most significant challenge. ORPI helps to identify these poor ovarian responders to tailor the stimulation protocol as per patients response and hence achieve individualization in ovarian stimulation-a new era in IVF.

In the study by Oliveira et al in 2013, they suggested several stimulation regimens grounded on the results of the ORPI. Following table shows deployment of the ovarian stimulation protocol and doses of folliclestimulating hormone (FSH) in the groups categorised by ORPI.

ORPI values of $<0.2$ were shown to have the best sensitivity $(86 \%)$ and specificity $(89 \%)$ in predicting a poor ovarian responder. Similarly, an ORPI of $\geq 0.9$ was shown to have the best sensitivity (89\%) and specificity $(86 \%)$ in predicting a high ovarian responder. There is no conventional ovarian stimulation regimen universally useful for every single patient. Based on its predictive potential, the ORPI might be used as a tool in the individualised planning of the medication doses and/or ovarian stimulation regimens.

\section{METHODS}

In this retrospective study a total of 50 patients who underwent IVF at infertility clinic between 2016 and 2017 were enrolled.

\section{Inclusion criteria}

- $\quad$ Age $\leq 39$ yrs
- $\quad$ BMI 20-30

- Regular menstrual cycle

- Both ovaries present

- No h/o ovarian surgery

- No severe endometriosis

- No evidence of endocrine disorders.

\section{Exclusion criteria}

Presence of ovarian cysts as assessed by TVS.

\section{AMH measurement}

A venous sample for AMH measurement was taken AMH can be measured at any time of menstrual cycle AMH is produced by granulose cells of preantral and small antral follicles. The serum level of AMH in females with normal cycles declines with age and becomes undetectable after menopause unlike other markers

Poor response is associated with serum AMH $<1 \mathrm{ng} / \mathrm{ml}$, normal response with $1-4 \mathrm{ng} / \mathrm{ml}$ and high response with $>\mathrm{ng} / \mathrm{ml}$

\section{Antral follicle count}

Using TVS in early follicular phase all ovarian follicles2$10 \mathrm{~mm}$ were counted and total for both ovaries was called as the basal AFC. The AFC correlates well with chronological age in normal fertile woman and appears to reflect what remains of the primordial follicular pool.A total AFC of $<4$ is predictive of poor ovarian response and higher cancellation rates with IVF.

\section{Calculation of ovarian response prediction index}

The ORPI was defined by the following equation

$\mathrm{ORPI}=(\mathrm{AMH} \times \mathrm{AFC}) /$ patient age

This equation is based on previous evaluations that found that ovarian response to stimulation had positive correlations with AMH levels and number of antral follicles and was negatively correlated with patient's age.

Notably, the calculated value of the ORPI in the study was not influenced by the protocol choice for the induction of ovulation or the doses of gonadotropin.

\section{Ovarian stimulation protocol}

The controlled ovarian stimulation (COS) protocols consist of a long GnRH agonist or a multidose $\mathrm{GnRH}$ antagonist protocol individualised for each patient.

The GnRH agonist protocol was performed using a $1 \mathrm{mg}$ /day dose of long acting GnRH agonist (leuprolide acetate) during the 14 days of luteal phase of the previous menstrual cycle for downregulation Then ovarian 
stimulation was started by daily injection of recombinant follicle stimulating hormone $(\mathrm{rFSH})$ with a starting dose specific for each individual case, according to the patient's age, BMI, ovarian reserve, and AFC.

\section{Endpoints}

In the present study, the overall predictive performance of age, AFC, and ORPI was calculated for the retrieval of $\geq 4$ M II oocytes, $\geq 15$ oocytes were accepted as the criterion of excessive response.

The collection of $\geq \mathrm{M}$ II oocytes will be classified as adequate response and $\geq 15$ oocytes will be classified as excessive ovarian response.

\section{RESULTS}

When there is an average (or high) number of antral follicles, we tend to get a "good" response with many mature follicles. We tend to get a good number of eggs at retrieval in these cases. Pregnancy rates are higher than average. When there are few antral follicles, we tend to get a poor response with few mature follicles. Cancellation of an IVF cycle is much more common when there is a low antral count. Pregnancy rates are lower overall in this group.

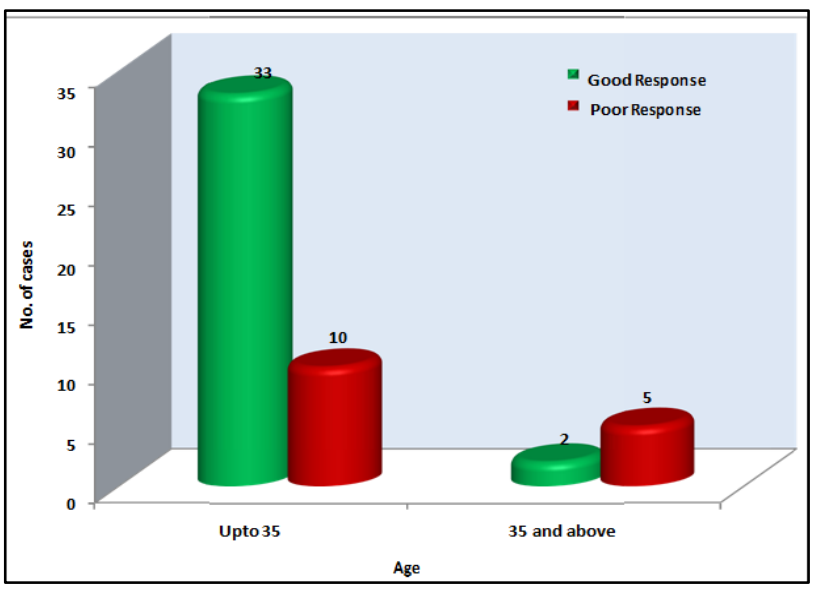

Figure 1: Correlation of age and IVF outcome.

The reduction in success rates is more pronounced in women over 35 years old. ${ }^{6}$ There is mounting evidence to support the use of $\mathrm{AMH}$ as a screening test for poor ovarian response, but more data is needed. There is emerging evidence to suggest that a low AMH level (for e.g. undetectable $\mathrm{AMH}$ ) has high specificity as a screen for poor ovarian response but insufficient evidence to suggest its use to screen for fail-ure to conceive.

There is fair evidence to support that a low antral follicle count has moderate to high specificity as a screening test for poor ovarian response and insufficient evidence to support the use of AFC as a screening test for failure to conceive.
Table 1: Correlation of AFC and IVF outcome.

\begin{tabular}{|lll|}
\hline AFC & Good response & Poor response \\
\hline upto 4 & 6 & 6 \\
\hline $5-20$ & 13 & 25 \\
\hline
\end{tabular}

In present study AFC had a sensitivity of $34.2 \%$ and a specificity of $50 \%$ in predicting ovarian response.

Table 2: Correlation of AMH and IVF outcome.

\begin{tabular}{|lll|}
\hline AMIH & Good response & Poor response \\
\hline upto 3 & 6 & 8 \\
\hline 3 and above & 34 & 2 \\
\hline
\end{tabular}

In present study $\mathrm{AMH}$ predicted ovarian response with a sensitivity of $94.4 \%$ and specificity of $57.1 \%$.

Table 3: ORPI and IVF outcome.

\begin{tabular}{|ll|}
\hline ORPI & No. of oocytes retrieved \\
\hline$<0.5$ & Upto 5 \\
\hline $0.6-0.9$ & $6-14$ \\
\hline$>0.9$ & 15 and above \\
\hline
\end{tabular}

In present study ORPI predicted ovarian response with a sensitivity of $88.23 \%$ and specificity of $81.25 \%$.

\section{DISCUSSION}

\section{Correlation of age and IVF outcome}

In present study Sensitivity of age (as a parameter to suggest good ovarian response) $=76.7 \%$; Specificity $=$ $71.4 \% ; \mathrm{PPV}=94.2 \% ; \mathrm{NPV}=33.33 \%$. Ernest Hung et al who studied effect of age on hormonal and USG markers of ovarian reserve in Chinese women and found AFC shows best correlation with age (Figure 1). ${ }^{5}$

Broeckman et al studied ovarian aging and found variability of ovarian aging among women which was evident from large variation of age at menopause. So, the identification of women with severely decreased ovarian reserve for their age is clinically relevant. ${ }^{6}$

With increasing age, ovarian reserve diminishes and spontaneous fecundity rate as well as success rates in IVF programs decline. The age related decrease in fertility is primarily due to oocyte senescence rather than due to poor endometrial receptivity, as suggested by the observation of high pregnancy outcome in oocyte donation programs by Sauer et al.

\section{Correlation of AFC and IVF outcome}

In present study AFC had sensitivity to predict ovarian response) of $34.2 \%$, specificity $=50 \%$, PPV $68.4 \%$, NPV $19.3 \%$. Peter et al found in their study that when the number of antral follicles is intermediate, the response is 
not as predictable. In most cases the response is intermediate. However, we could also have either a low or a good response when the antral counts are intermediate. Pregnancy rates are pretty good overall in this group.

\section{Correlation of AMH and IVF outcome}

In present study Sensitivity of AMH to predict ovarian response $=94.4 \%$, Specificity $=57.1 \%, \mathrm{PPV}=85 \%$, $\mathrm{NPV}=80 \%$.

In Gomez et al studied the influence of AMH on IVF success and concluded that AMH is a useful parameter that should be measured before performing an IVF/ICSI treatment. In younger patients, AMH levels do not predict pregnancy outcomes. In patients older than 36 years, AMH can be used as a prognostic factor. Even when a woman's AMH levels are too low to be detected, she still has an acceptable chance of becoming pregnant. ${ }^{7}$

\section{ORPI and IVF outcome}

Sensitivity of ORPI in predicting ovarian response $=88.23 \% \quad$ Specificity $=81.25 \% \quad \mathrm{PPV}=76.47 \%$. This value of ORPI of 0.5 was obtained from the work of Oliviera et al.

Oliviera et al established the ORPI and gave the following values. From Table 3 ORPI seems to predict ovarian response better than AFC and AMH alone.

\section{Incidence of OHSS}

Table 4: Incidence of OHSS.

\begin{tabular}{|lllll|}
$\begin{array}{l}\text { Patient } \\
\text { name }\end{array}$ & $\begin{array}{l}\text { OHSS (No. of } \\
\text { oocytes } \\
\text { retrieved) }\end{array}$ & ORPI & AMIH & AFC \\
\hline 1 & 16 & 2.90 & 6 & $16(\mathrm{pcos})$ \\
\hline 2 & 15 & 2.5 & 6.03 & 10 \\
\hline 3 & 20 & 4.62 & 9.91 & $14(\mathrm{pcos})$ \\
\hline 4 & 26 & 4.01 & 9.10 & $15(\mathrm{pcos})$ \\
\hline 5 & 28 & 8 & 8.4 & $20(\mathrm{pcos})$ \\
\hline 6 & 24 & 8 & 12.8 & $15(\mathrm{pcos})$ \\
\hline 7 & 16 & 4.29 & 5.73 & $21 \mathrm{pcos}$ \\
\hline
\end{tabular}

In view of all these studies, if we take $\mathrm{AMH}>3.5$ as predictor of OHSS, all 7 OHSS patients had AMH values more than 3.5. But there were 6 more patients in our study with AMH values mare than 3.5 but who did not have OHSS.

With reference to prior studies, if we take AFC >20 as predictor of OHSS, only 2 out of our 7 patients with OHSS fulfil these criteria. However, none of the rest of our patients had AFC more than 20.
Not much work has been done to evaluate ORPI as an index to predict ovarian response, work done by Oleiviera et al, suggested ORPI $>2.5$ as predictor of OHSS. In present study all 7 OHSS patients had ORPI more than 2.5 and none of the patients without OHSS had value of ORPI more than 2.5 . $^{7}$

\section{CONCLUSION}

Because no single measure of ovarian reserve has $100 \%$ sensitivity and specificity, biochemical and imaging measures have been combined in an effort to improve test characteristics.

Available evidence concerning the performance of ovarian reserve tests is limited by small sample sizes, heterogeneity among study design, analyses and outcomes, and by the lack of validated results. The design of published studies must be examined carefully before applying the results in clinical practice A number of different "ovarian reserve tests" have been devised as screening tests to help predict success with IVF.

Ideally, the optimal screening test should be reproducible (low inter- and intra-cycle variability) and exhibit high specificity to minimize the risk of incorrectly categorizing women with normal ovarian reserve as having decreased ovarian reserve. A screening test itself cannot diagnose decreased ovarian reserve.

\section{Funding: No funding sources}

Conflict of interest: None declared

Ethical approval: The study was approved by the Institutional Ethics Committee

\section{REFERENCES}

1. Makkar G, Ng EH, Yeung WS, Ho PC. Excessive ovarian response is associated with increased expression of interleukin-2 in the periimplantation endometrium. Fertil Steril. 2009 Apr 1;91(4):114551.

2. Broekmans FJ, Kwee J, Hendriks DJ, Mol BW, Lambalk CB. A systematic review of tests predicting ovarian reserve and IVF out-come. Hum Reprod Update. 2006;12:685-718.

3. Sauer MV, Paulson RJ, Lobo RA. A preliminary report on oocyte donation extending reproductive potential to women over 40. $\mathrm{N}$ Engl $\mathrm{J}$ Med. 2000;323:1157-60.

4. Peters H, Byskov, AG, Himelstein-Graw R, Faber M. Follicular growth (the basic event in the mouse and human ovary). J Reprod Fertil. 2000;45:559-66.

5. Muttukrishna S, McGarrigle H, Wakim R, Khadum I, Ranieri DM, Serhal P. Antral follicle count, antimullerian hormone and inhibin B: predictors of ovarian response in assisted reproductive technology? BJOG. 2005;112:1384-90. 
6. Singh M, Malik E. AMH as predictive marker of ovarian response in ART. Human Reprod. 2012;43:56.

7. Aflatoonian A, Oskouian H, Ahmadi S, Oskouian L. Prediction of high ovarian response to controlled ovarian hyperstimulation: anti-Mullerian hormone versus small antral follicle count (2-6 mm). J Assist Reprod Genet. 2009 Jun;26(6):319-25.

Cite this article as: Tak A, Chauhan M, Tak B.

Prognosticating ovarian reserve by the new ovarian response prediction index. Int J Reprod Contracept Obstet Gynecol 2018;7:1196-200. 